\title{
Cytocidal amino acid starvation of Saccharomyces cerevisiae and Candida albicans acetolactate synthase (ilv2 $\Delta$ ) mutants is influenced by the carbon source and rapamycin
}

\author{
Correspondence \\ John H. McCusker \\ mccus001@mc.duke.edu
}

Received 7 September 2009

Revised 4 November 2009

Accepted 10 December 2009

\author{
Joanne M. Kingsbury and John H. McCusker
}

Department of Molecular Genetics and Microbiology, Box 3020, Duke University Medical Center, Durham, NC 27710, USA

The isoleucine and valine biosynthetic enzyme acetolactate synthase (Ilv2p) is an attractive antifungal drug target, since the isoleucine and valine biosynthetic pathway is not present in mammals, Saccharomyces cerevisiae ilv2 $\Delta$ mutants do not survive in vivo, Cryptococcus neoformans ilv2 mutants are avirulent, and both S. cerevisiae and Cr. neoformans ilv2 mutants die upon isoleucine and valine starvation. To further explore the potential of llv $2 p$ as an antifungal drug target, we disrupted Candida albicans ILV2, and demonstrated that Ca. albicans ilv2 $\Delta$ mutants were significantly attenuated in virulence, and were also profoundly starvation-cidal, with a greater than 100 -fold reduction in viability after only $4 \mathrm{~h}$ of isoleucine and valine starvation. As fungicidal starvation would be advantageous for drug design, we explored the basis of the starvation-cidal phenotype in both S. cerevisiae and Ca. albicans ilv2 $\Delta$ mutants. Since the mutation of ILV1, required for the first step of isoleucine biosynthesis, did not suppress the ilv2 $\Delta$ starvation-cidal defects in either species, the cidal phenotype was not due to $\alpha$-ketobutyrate accumulation. We found that starvation for isoleucine alone was more deleterious in Ca. albicans than in $S$. cerevisiae, and starvation for valine was more deleterious than for isoleucine in both species. Interestingly, while the target of rapamycin (TOR) pathway inhibitor rapamycin further reduced $S$. cerevisiae ilv2 $\Delta$ starvation viability, it increased Ca. albicans ilv $1 \Delta$ and ilv $2 \Delta$ viability. Furthermore, the recovery from starvation was dependent on the carbon source present during recovery for $S$. cerevisiae ilv2 $\Delta$ mutants, reminiscent of isoleucine and valine starvation inducing a viable but nonculturable-like state in this species, while Ca. albicans ilv1 $\Delta$ and ilv2 $\Delta$ viability was influenced by the carbon source present during starvation, supporting a role for glucose wasting in the $\mathrm{Ca}$. albicans cidal phenotype.

\section{INTRODUCTION}

Due to the high level of conserved pathways between fungi and mammals, effective antifungal therapy relies upon drugs from only three main drug classes, the polyenes, azoles and echinocandins, which target only two cellular components, the cell membrane and cell wall (Tkacz \& DiDomenico, 2001). In addition to the cell wall, another fundamental difference between fungi and mammals is the ability of fungi to synthesize all amino acids, while

Abbreviations: FRT, FLP recombination target; Nat, nourseothricin; TOR pathway, target of rapamycin pathway.

Two supplementary figures, showing the construction of Candida albicans deletion mutants, Southern hybridization analysis confirming gene replacements and growth of $S$. cerevisiae and Ca. albicans wildtype, ilv $1 \Delta$ and ilv2 $\Delta$ strains in YPD and SD + isoleucine + valine media, and a supplementary table, listing primers used in this study, are available with the online version of this paper. mammals must acquire many amino acids, such as isoleucine and valine, in their diet. Certain in vivo environments occupied by fungal pathogens are likely to be limiting in amino acids, as serum amino acid concentrations are low (Crispens, 1975; Cynober, 2002), genes for the biosynthesis and transport of various amino acids and the general control regulator Gcn4p are induced upon exposure of fungi to neutrophils, macrophages or blood (Fradin et al., 2003, 2005; Lorenz et al., 2004), and various amino acid auxotrophs are unable to survive in vivo and/or are avirulent (Goldstein \& McCusker, 2001; Kingsbury et al., 2004a, b, 2006; Kingsbury \& McCusker, 2008; Liebmann et al., 2004; Nazi et al., 2007; Pascon et al., 2004; Yang et al., 2002). Therefore, various amino acid biosynthetic enzymes offer an attractive alternative class of antifungal targets.

Since many fungal infections occur in immunocompromised patients, and fungistatic drugs require a healthy 
immune system to clear the infection so that it does not recrudesce upon drug removal, targeting biosynthetic enzymes for which the resulting auxotrophy is fungicidal rather than fungistatic is advantageous. The degree of viability loss during starvation is nutrient-dependent; for example, while starvation for methionine, phosphate or sulfate is generally cytostatic (Barclay \& Little, 1977; Boer et al., 2008; Kingsbury et al., 2004a; Unger \& Hartwell, 1976), starvation for leucine or uracil is minimally cytocidal [50\% reduction after 2 days (Boer et al., 2008)], while inositol starvation is highly cytocidal [ $\geqslant 99 \%$ reduction after 1 day (Culbertson \& Henry, 1975; Henry et al., 1975)]. Viability during starvation has been shown to correlate with how rapidly the cell cycle arrests upon starvation, with methionine-, phosphate- and sulfate-starved cells undergoing a prompt and uniform arrest in the $G_{1} / G_{0}$ (unbudded) stage (Saldanha et al., 2004; Unger \& Hartwell, 1976), while leucine-starved cells do not undergo uniform arrest (Saldanha et al., 2004), and waste the excess glucose present (Brauer et al., 2008). The degree of viability reduction is also dependent on the carbon source available during starvation and the genetic background (Boer et al., 2008). Finally, the starvation-cidal phenotype may be contingent on which biosynthetic enzyme of the particular pathway is inhibited or mutated; for example, Cryptococcus neoformans (and Saccharomyces cerevisiae) met3 mutants are cytostatic for methionine starvation, while Cr. neoformans met6 mutants, which accumulate the toxic intermediate homocysteine, are cytocidal (Pascon et al., 2004).

Enzymes of the isoleucine and valine biosynthetic pathway such as acetohydroxy acid synthase (Ilv2p) are of particular interest from the perspective of antifungal drug targets,

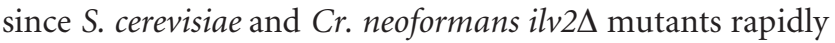

lose viability during isoleucine and valine starvation [90\% reduction after 1 day (Kingsbury et al., 2004a)], and do not survive in vivo and/or are avirulent (Kingsbury et al., 2004a, 2006). The first step of isoleucine biosynthesis (reviewed by Chipman et al., 1998; Fig. 1) involves the deamination of threonine to $\alpha$-ketobutyrate by threonine deaminase (Ilv1p). The second step, catalysed by Ilv2p, combines $\alpha$-ketobutyrate and pyruvate to yield $\alpha$-acetolactate. Ilv $2 \mathrm{p}$ also converts two pyruvate molecules to $\alpha$-aceto$\alpha$-hydroxybutyrate, an intermediate in valine and leucine biosynthesis. The absence of acetolactate synthase activity in bacteria results in elevated levels of $\alpha$-ketobutyrate, which has been hypothesized to inhibit growth by interfering with utilization of glucose (LaRossa \& Van Dyk, 1987; LaRossa et al., 1987; Van Dyk et al., 1987). Therefore, $\alpha$-ketobutyrate accumulation may explain the deleterious phenotypes of fungal ilv $2 \Delta$ mutants, such as the starvation-cidal phenotype.

Since Cr. neoformans and S. cerevisiae ilv $2 \Delta$ mutants are avirulent and/or unable to survive in vivo (Kingsbury et al., 2004a, 2006), and die upon starvation for isoleucine and valine, we further investigated the potential of acetolactate synthase as a drug target in the highly clinically relevant pathogen Candida albicans. Importantly, we found that $\mathrm{Ca}$. albicans ilv2 $2 \Delta$ mutants were significantly attenuated in virulence, and undergo a dramatic decline in viability upon isoleucine and/or valine starvation. By looking at the starvation phenotypes of other isoleucine-valine pathway mutants, we tested the two following hypotheses to explain the basis of the starvation-cidal effect in both S. cerevisiae and Ca. albicans: first, that death is due to toxic $\alpha$ ketobutyrate accumulation; and second, that death is a consequence of isoleucine, leucine and/or valine starvation.

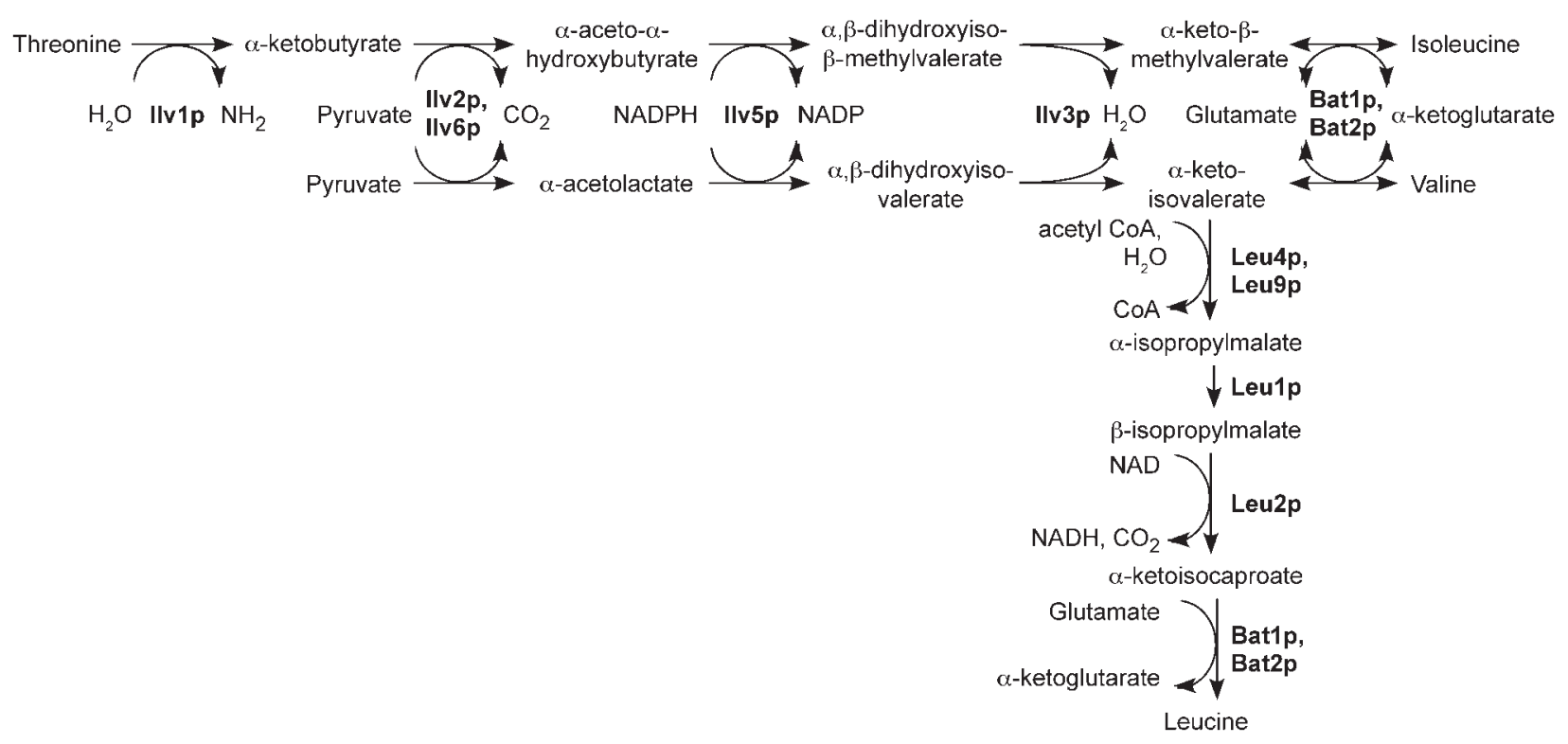

Fig. 1. Isoleucine, valine and leucine biosynthetic pathways. 
We exclude the $\alpha$-ketobutyrate hypothesis, and demonstrate that the cidal effect is due to starvation for isoleucine and/or valine, with valine starvation being more deleterious. We examined the effects of rapamycin on starvation, which we show to be deleterious for S. cerevisiae survival, yet beneficial for Ca. albicans survival. We further determine that the extent of loss of viability is governed by the carbon source present during starvation recovery for $S$. cerevisiae, and the carbon source present during starvation for Ca. albicans.

\section{METHODS}

Strains, media and growth conditions. Ca. albicans strains used in this study were isogenic with strain SC5314 (Gillum et al., 1984), and S. cerevisiae strains were isogenic with S288c or YJM145 (McCusker et al., 1994) (Table 1). Standard yeast culture media including yeast extract peptone dextrose (YPD), yeast extract peptone ethanol glycerol (YPEG) and synthetic dextrose (SD) were prepared as described previously (Ito-Harashima et al., 2002; Sherman et al., 1974). SD (proline) contained $1 \%(\mathrm{w} / \mathrm{v})$ proline instead of ammonium sulfate. Yeast extract peptone maltose [YP(maltose)] was prepared as for YPD, expect that maltose $(2 \%$, w/v) replaced glucose. Synthetic ethanol glycerol (SEG) liquid media contained succinic acid $(1 \%, \mathrm{w} / \mathrm{v})$, glycerol $(2 \%, \mathrm{v} / \mathrm{v})$ (mixed, adjusted to pH 5.5 with potassium hydroxide), yeast nitrogen base with ammonium sulfate $(0.67 \%, \mathrm{w} / \mathrm{v})$ and ethanol $(2.5 \%, \mathrm{v} / \mathrm{v})$, and was filter-sterilized. Where specified, media were supplemented with isoleucine $(0.23 \mathrm{mM})$, valine $(1.28 \mathrm{mM})$ or leucine $(0.46 \mathrm{mM})$, nourseothricin (Nat; $100 \mu \mathrm{g} \mathrm{ml}{ }^{-1}$ for S. cerevisiae selection, $200 \mu \mathrm{g}$ $\mathrm{ml}^{-1}$ for Ca. albicans selection; Hans Knöll Institute für NaturstoffForschung), hygromycin B (300 $\mu \mathrm{g} \mathrm{ml}^{-1}$, Calbiochem) or geneticin (200 $\mu \mathrm{g} \mathrm{ml}^{-1}$, Life Technologies).

Strain construction. S. cerevisiae genes were replaced with the natMX4, kanMX4, hphMX4 or loxP-kanMX4-loxP cassettes by PCRmediated gene deletion, as described previously (Goldstein \& McCusker, 1999; Guldener et al., 1996; Wach et al., 1994). To construct strains containing multiple deletions, separate strains containing deletions with different drug markers were crossed, crosses were sporulated and dissected, and segregants with the appropriate genotype were selected for further analysis. Gene deletions were confirmed by PCR and by acquisition or loss of a phenotype.

Ca. albicans genes were replaced with the SAT1 flipper cassette from plasmid pSFS2A (Reuss et al., 2004) using a similar PCR-mediated disruption strategy, in which the SAT1 flipper cassette was amplified using primers that contained at their $5^{\prime}$ ends $60 \mathrm{bp}$ of sequence homologous to the region immediately flanking the gene of interest. Strains were transformed with the gene-targeting PCR product by electroporation (Reuss et al., 2004), and Nat-resistant transformants were purified and verified by PCR analysis. To induce FLP-mediated excision of the SAT1 cassette, transformants were grown for $2 \mathrm{~h}$ in $\mathrm{YP}$ (maltose), leaving an FLP recombination target (FRT) site. Natsensitive $+/ \Delta$ strains then underwent a second round of transformation to disrupt the second allele. To reintroduce the wild-type gene, homozygous disruption strains were transformed with the wild-type gene of interest, and amplified using primers homologous to

Table 1. Strains used in this study

\begin{tabular}{|c|c|c|}
\hline Strain & Genotype $^{*}$ & Reference or source \\
\hline \multicolumn{3}{|c|}{ Ca. albicans } \\
\hline SC5314 & Wild-type & Gillum et al. (1984) \\
\hline CJK23 & ILV2/ilv2 $:$ : FRT & This study \\
\hline CJK27 & 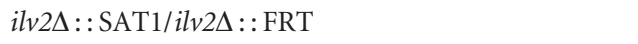 & This study \\
\hline CJK80 & MET2/met $2 \Delta::$ SAT1 & This study \\
\hline CJK103 & met $2 \Delta:: \mathrm{SAT} 1 /$ met $2 \Delta:: \mathrm{FRT}$ & This study \\
\hline CJK131 & 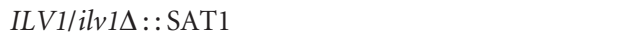 & This study \\
\hline CJK132 & 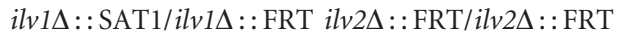 & This study \\
\hline CJK133 & ILV2/ilv2A:: SAT1 (ILV2-complemented strain) & This study \\
\hline CJK138 & ILV1/ilv1 $\Delta:$ :FRT & This study \\
\hline CJK140 & $i l v 1 \Delta:: \mathrm{SAT} 1 / i l v 1 \Delta:: \mathrm{FRT}$ & This study \\
\hline \multicolumn{3}{|c|}{ S. cerevisiae } \\
\hline S3783 & 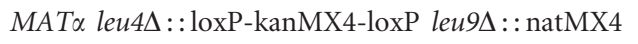 & This study \\
\hline S3807 & MATa leu2A: : hphMX4 & This study \\
\hline YJK564 & MATa/MAT $\alpha$ met $2 \Delta::$ natMX $4 /$ met $2 \Delta::$ natMX 4 & This study \\
\hline YJK2484 & MATa ilv1s: : kanMX4 & This study \\
\hline YJK2486 & MATa ilv2 2 : : natMX4 & This study \\
\hline YJK2487 & MATa ilv1s: : kanMX4 ilv2 $\Delta:$ : natMX4 & This study \\
\hline
\end{tabular}

${ }^{*}$ All Ca. albicans strains were isogenic with SC5314 and all S. cerevisiae strains were isogenic with S288c, except YJK564, which was isogenic with YJM145 (McCusker et al., 1994). 
sequences upstream and downstream of the deleted region. Transformants in which the wild-type allele had replaced a disrupted allele were chosen; thus, the complementing gene was expressed from its original chromosomal location. All disruptions and mutant complementations were verified by PCR, phenotype where available, and Southern hybridization analysis (Supplementary Fig. S1).

All primers used are listed in Supplementary Table S1.

Manipulation of DNA. S. cerevisiae and Ca. albicans genomic DNA was extracted as described previously (Hoffman \& Winston, 1987). For Southern hybridization analyses, Ca. albicans DNA $(2 \mu \mathrm{g})$ was digested with various restriction enzymes, separated by gel electrophoresis in a $1 \%(\mathrm{w} / \mathrm{v})$ agarose gel, denatured and transferred to a nylon membrane (Roche), as described previously (Sambrook et al., 1989). Hybridization probes were PCR-amplified using the primer pairs JO784+JO787 (ILV1), JO515+JO518 (ILV2), and JO662 + JO663 (MET2). Probes were purified following agarose gel electrophoresis using the QIAquick Gel Extraction kit (Qiagen) and labelled with $\left[\alpha-{ }^{32} \mathrm{P}\right] \mathrm{dCTP}$ (Perkin-Elmer) using the Rediprime II Random Prime Labeling system (Amersham Biosciences), as described by the manufacturer. Prehybridizations and hybridizations were carried out in ULTRAhyb buffer (Ambion), membranes were washed according to manufacturer's instructions, and hybridized bands were visualized using a Typhoon 9200 Variable Mode imager (Molecular Dynamics).

Mouse infections. The virulence of Ca. albicans strains was tested by injecting 6-week-old male CD-1 mice with $1 \times 10^{6}$ cells suspended in sterile PBS, via the lateral tail vein. Typically, 10 mice were infected per Ca. albicans strain tested. Mice were fed ad libitum for the course of the experiment. Mice were observed and weighed twice daily, and animals that appeared moribund (judged by $>15 \%$ loss of body weight, lethargy, or being unable to access food) were euthanized. Mice that remained healthy after 28 days were euthanized, and their kidneys, liver and spleen were recovered and homogenized in $3 \mathrm{ml}$ $\mathrm{PBS}+$ streptomycin + ampicillin. The entire spleen and $250 \mu \mathrm{l}$ of each liver and kidney homogenate were plated on YPD + Nat to ascertain whether the infection had been cleared. Mouse survival data were analysed using the Kaplan-Meier test. Mouse experiments met with institutional guidelines and were approved by the Institutional Animal Care and Use Committee.

Starvation assays. To assess the ability of auxotrophic strains to survive amino acid starvation, overnight cultures grown in YPD or YPEG were pelleted, washed twice in sterile distilled water, and added to $5 \mathrm{ml} \mathrm{SD}$ or SEG to a concentration of approximately $10^{7}$ cells $\mathrm{ml}^{-1}$. Cultures were incubated at $30{ }^{\circ} \mathrm{C}$ with aeration, and at various time points, aliquots were removed, serially diluted and plated to YPD or YPEG to determine viable c.f.u. Freshly poured ( $<2$ weeks) YPD plates were used to circumvent as much as possible the considerable variation observed for ilv2 $2 \Delta$ mutant starvation recovery on different batches of YPD. For approximate numbers, $5 \mu$ volumes of 10 -fold dilutions were plated, while $100 \mu \mathrm{l}$ volumes were plated for more exact estimations; experiments were typically repeated in triplicate. S. cerevisiae colonies were counted after 3 days of incubation at $30{ }^{\circ} \mathrm{C}$ on YPD plates or 4 days of incubation on YPEG, and $\mathrm{Ca}$. albicans colonies were counted after 2 days of incubation on YPD or 3 days of incubation on YPEG.

$\boldsymbol{\alpha}$-Ketobutyrate inhibition assay. To determine the level of $\alpha$ ketobutyrate inhibition, strains were grown overnight in YPD, washed twice in water, and resuspended to a concentration of approximately $1-2 \times 10^{3}$ c.f.u. $\mathrm{ml}^{-1}$ in YPD. Volumes of $90 \mu \mathrm{l}$ of cells were added to $10 \mu$ of a twofold dilution series of $\alpha$-ketobutyrate in flat-bottomed microtitre plate (Corning) wells. Assays were performed in triplicate and incubated at $30{ }^{\circ} \mathrm{C}$ for $1-2$ days.
Growth assays. To determine the effect of isoleucine and valine auxotrophy on growth, wild-type, ilv $1 \Delta$ and $i l v 2 \Delta$ strains were grown overnight in YPD, washed twice in water, and resuspended in YPD or $\mathrm{SD}+$ isoleucine + valine media to $\mathrm{OD}_{600} 0.05$. One hundred microlitre volumes of strains were incubated in 96-well microtitre plates (Corning) at $30{ }^{\circ} \mathrm{C}$, and $\mathrm{OD}_{600}$ readings were taken half-hourly using an automated Tecan Sunrise absorbance reader. Experiments were performed in triplicate.

\section{RESULTS}

\section{Ca. albicans ilv1 $\Delta$ and ilv2 $\Delta$ mutants have attenuated virulence}

Since Cr. neoformans ilv2s mutants are avirulent (Kingsbury et al., 2004a) and S. cerevisiae ilv2 $2 \Delta$ mutants do not survive in vivo (Kingsbury et al., 2006), we constructed and tested the virulence in mice of $\mathrm{Ca}$. albicans

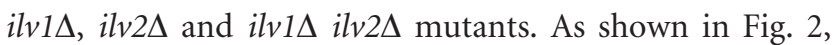
compared with the wild-type $(2.6 \pm 0.8$ days, $P=0.0254)$ and ILV1-complemented strain (CJK142; $1.6 \pm 1.3$ days, $P=0.0008$ ), mice infected with the ilv1 $\Delta$ mutant (CJK140) survived longer on average ( $6.6 \pm 5.5$ days). Therefore, isoleucine auxotrophy has a modest effect on Ca. albicans virulence.

Relative to ilv1 $\Delta$ mutants, the ilv2 $\Delta$ mutant (CJK27) was far more attenuated in virulence, with $73 \%$ of mice still surviving after 28 days; $P$ values were $<0.0029$ and $<0.0012$ compared with the wild-type and ILV2-complemented strains, respectively. Examination of the kidneys, liver and spleen from the eight mice still surviving after 28 days revealed the presence of viable ilv $2 \Delta$ mutants in the kidneys of six mice; thus, two mice had cleared the infection $\left(<22\right.$ c.f.u. $\mathrm{g}^{-1}$ of tissue). Therefore, isoleucine auxotrophy combined with valine auxotrophy has a more detrimental effect on Ca. albicans virulence than isoleucine auxotrophy alone.

The ilv1s ilv2s mutant, CJK132, was similarly shown to be significantly attenuated in virulence $(P<0.0004$ compared with the wild-type), with $80 \%$ of the mice still surviving after 28 days, of which, three of eight had also cleared the infection. Since Ilvlp is required for the biosynthesis of the intermediate $\alpha$-ketobutyrate, and the ilv1 $1 \Delta$ disruption did not suppress the attenuated virulence of the ilv2 $\Delta$ mutant, $\alpha$-ketobutyrate accumulation plays no role in the greatly attenuated virulence of the ilv $2 \Delta$ mutant.

\section{S. cerevisiae and Ca. albicans ilv2 $\Delta$ mutants die rapidly upon isoleucine and valine starvation}

Since S. cerevisiae and Cr. neoformans ilv2 mutants are avirulent and die upon isoleucine and valine starvation (Kingsbury et al., 2004a), survival during amino acid starvation is likely to be important in vivo. Therefore, the ability of S. cerevisiae and Ca. albicans ilv2 $2 \Delta$ mutants to survive isoleucine and valine starvation was compared. Averaged over three separate experiments, the S. cerevisiae 


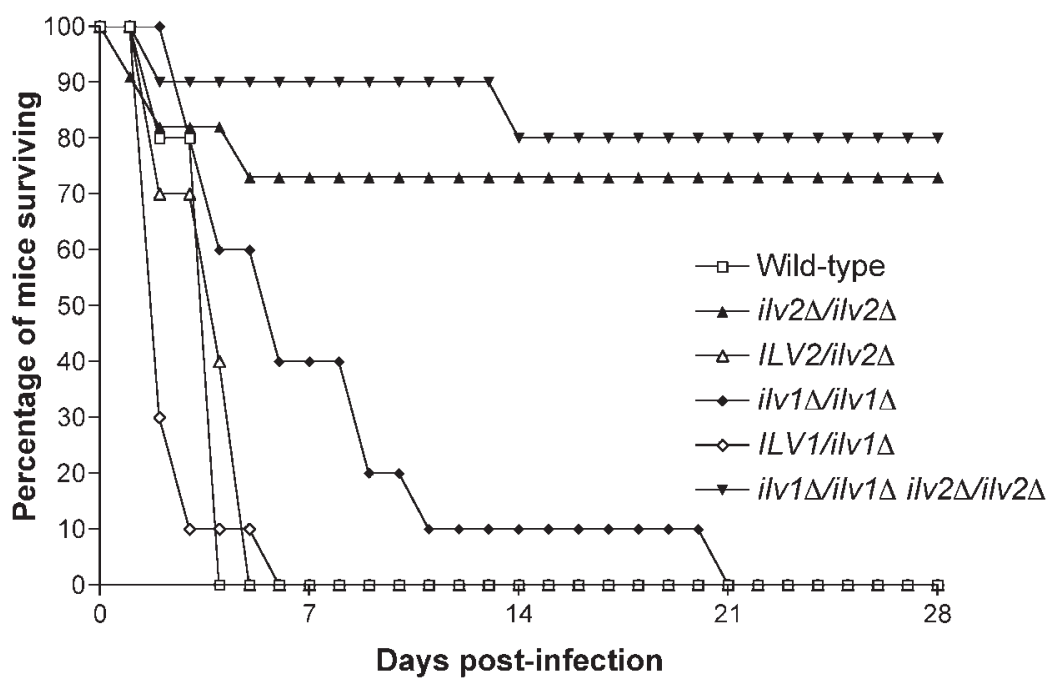

Fig. 2. Virulence phenotypes of Ca. albicans isoleucine and valine biosynthetic mutants. Strains included SC5314 (wild-type), CJK27

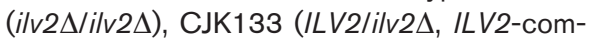

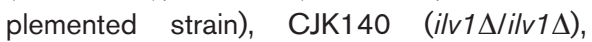
CJK142 (ILV1/ilv1A, ILV1-complemented

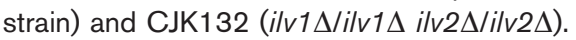

ilv2 $\Delta$ mutant (YJK2486) was recovered at approximately 13 -fold reduced levels after $24 \mathrm{~h}$ of amino acid starvation (Fig. 3a), similar to previous findings (Kingsbury et al., 2004a). The Ca. albicans ilv2s mutant (CJK27) underwent a substantially more dramatic decline in viability upon incubation in SD medium, with an average 167-fold reduction after only $4 \mathrm{~h}$, and further decreasing to a 658fold reduction after $8 \mathrm{~h}$, and a 2320-fold reduction after $24 \mathrm{~h}$ (Fig. 4a). Microscopic examination revealed that cells were predominantly single or budded; thus, cell clumping was excluded as an explanation for reduced c.f.u. Therefore, Ca. albicans ilv $2 \Delta$ mutants are starvation-cidal like $S$. cerevisiae and $C r$. neoformans ilv $2 \Delta$ mutants, but to a significantly greater degree.

\section{The S. cerevisiae and Ca. albicans ilv2 $\Delta$ starvation-cidal phenotype is not due to $\alpha$ - ketobutyrate accumulation}

The ilv2s mutant fungicidal phenotype upon isoleucine and valine starvation may be due to toxicity caused by $\alpha$ ketobutyrate accumulation. If $\alpha$-ketobutyrate accumulation is toxic, ilv2s mutants would likely be hypersensitive to exogenously added $\alpha$-ketobutyrate. To test whether $\alpha$ ketobutyrate is actually transported by cells, we first assessed the ability of exogenous $\alpha$-ketobutyrate to satisfy the auxotrophy of $S$. cerevisiae and Ca. albicans ilv1s mutants (YJK2484 and CJK140), when added to a filter disk ( $50 \mu \mathrm{l}$ of $100 \mathrm{mg} \mathrm{ml}^{-1}$ ) on SD or SD (proline) plates to which the strains were applied. While the $\alpha$-ketobutyrate only minimally satisfied the $S$. cerevisiae ilv1s mutant auxotrophy, $\alpha$-ketobutyrate supported robust growth of the Ca. albicans ilv1s mutant on both media types (data not shown). The ability of $\alpha$-ketobutyrate to inhibit growth was then determined for S. cerevisiae and Ca. albicans wildtype (S094 and SC5314), ilv2A (YJK2486 and CJK27), and ilv1s ilv2s (YJK2487 and CJK132) strains in YPD. Even at the highest concentration of $\alpha$-ketobutyrate $\left(10 \mathrm{mg} \mathrm{ml}^{-1}\right)$, no growth inhibition was observed for any strain, which argues against the hypothesis that $\alpha$-ketobutyrate is toxic in fungi.

To further test the $\alpha$-ketobutyrate accumulation hypothesis, we compared the amino acid starvation phenotypes

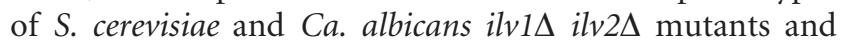
ilv2 $\Delta$ mutants. Viability following starvation of both the $S$. cerevisiae ilv1s ilv2s mutant (YJK2487) and the $C a$.

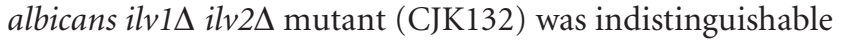
from that of the $S$. cerevisiae and $C a$. albicans ilv2 $\Delta$ mutants, respectively (Figs $3 \mathrm{a}$ and $4 \mathrm{a}$ ). Since disruption of ILV1 did not suppress the starvation-cidal phenotype of the ilv2 $2 \Delta$ mutants in either S. cerevisiae or Ca. albicans, $\alpha-$ ketobutyrate accumulation does not play a role in this phenotype.

\section{The ilv2 $\Delta$ mutant starvation-cidal phenotype is due to valine starvation in $\mathbf{S}$. cerevisiae, and multiple amino acid auxotrophies in $\mathbf{C a}$. albicans}

The ilv2 $2 \Delta$ mutants are auxotrophic for isoleucine, valine and leucine (Fig. 1). We therefore assessed various individual and combined contributions of starvation for isoleucine, valine or leucine to the starvation-cidal phenotype.

We first determined the effect of isoleucine starvation. If the cidal phenotype is due to isoleucine starvation alone, supplementation of media with isoleucine during starvation, which results in valine and leucine starvation alone, may enhance ilv $2 \Delta$ viability. However, neither S. cerevisiae nor $C a$. albicans ilv $2 \Delta$ mutant viability was improved by isoleucine addition (Figs $3 \mathrm{~b}$ and $4 \mathrm{~b}$ ). Since we observed considerably reduced growth of both $S$. cerevisiae and $\mathrm{Ca}$. albicans ilv1s mutants in SD medium supplemented with isoleucine (and valine) (Supplementary Fig. S2), the lack of effect of isoleucine supplementation could be attributed to poor isoleucine uptake by ilv $2 \Delta$ mutants. Alternatively, the 
(a)

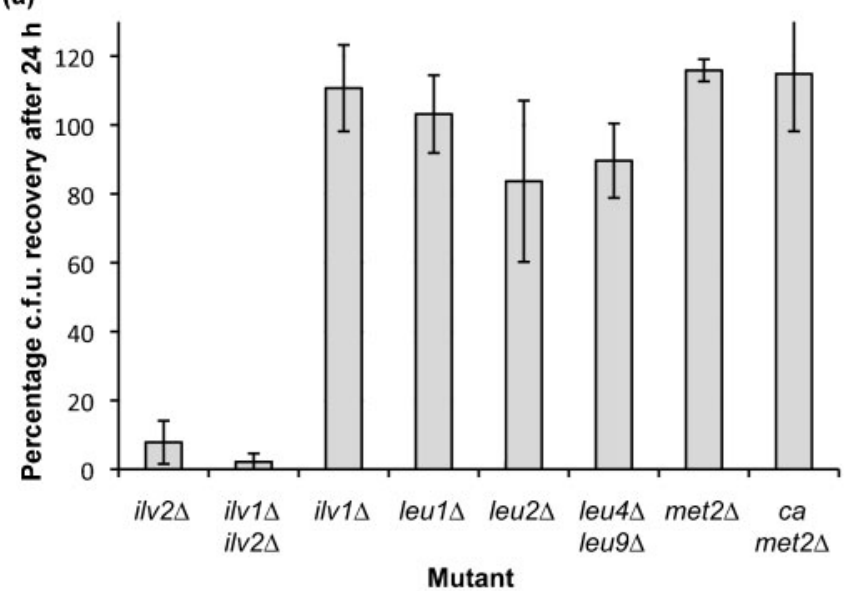

(c)

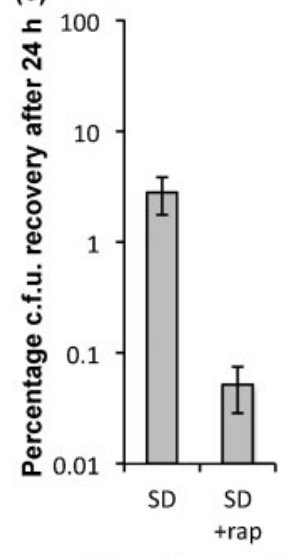

Starvation media

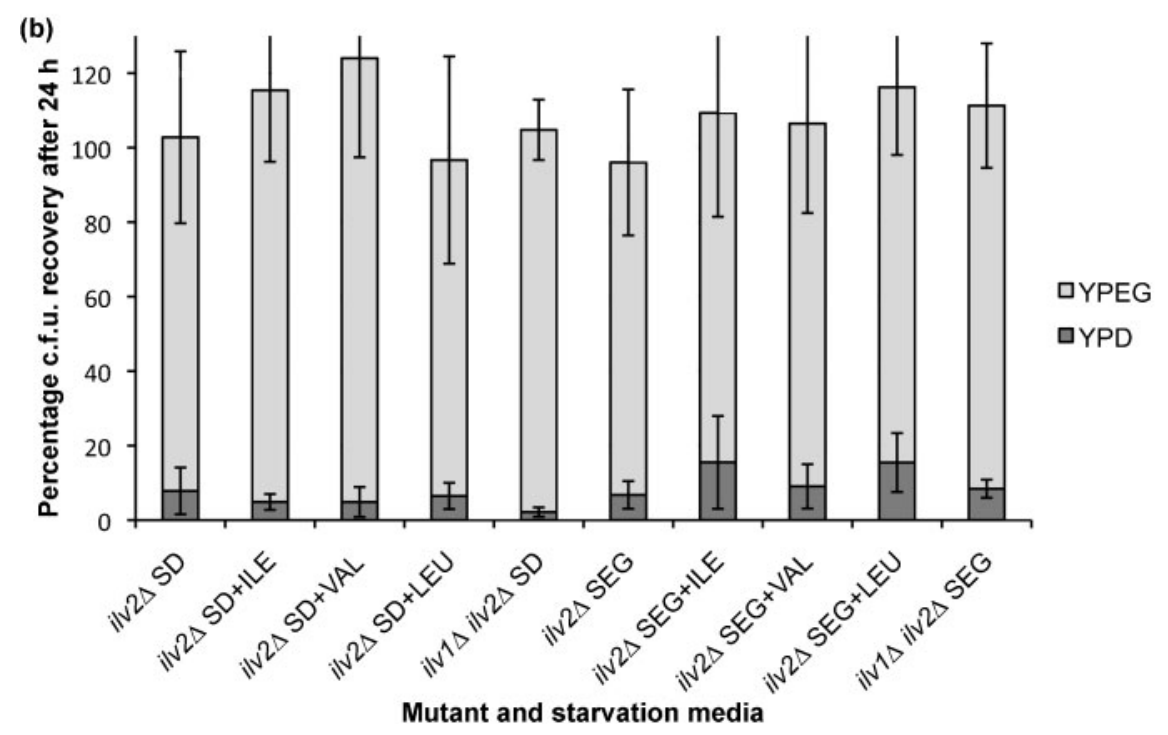

Fig. 3. (a) Recovery on YPD following starvation of S. cerevisiae (and Ca. albicans met2A) auxotrophs in SD. (b) Recovery on YPD and YPEG following starvation in SD or SEG \pm amino acid supplementation, as indicated. (c) Recovery of $S$. cerevisiae ilv2 $\Delta$ mutant on YPD following starvation in SD or SD + rapamycin (SD + rap). Strains included S. cerevisiae YJK2486 (ilv2 4 ),

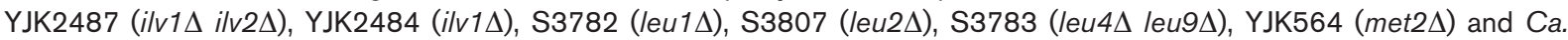
albicans CJK103 (ca met2A).

results may indicate that, in both species, valine and/or leucine starvation can account for all of the starvation-cidal phenotypes.

To further test whether isoleucine starvation is cidal, the viability following amino acid starvation was tested for $S$. cerevisiae and Ca. albicans ilv1s mutants (YJK2484 and CJK40, respectively), which are auxotrophic only for isoleucine. The $S$. cerevisiae ilv1s mutant viability remained unchanged following $24 \mathrm{~h}$ starvation (c.f.u. were, on average, $111 \%$ of initial levels; Fig. 3a). Therefore, isoleucine starvation alone is not cidal for S. cerevisiae and hence does not explain the ilv2 $\Delta$ cidal phenotype. In contrast, the Ca. albicans ilv $1 \Delta$ mutant reduced in viability over time, although to a lesser extent than the ilv2 $\Delta$ mutant, with an average 32 -fold reduction in viability from input levels after $4 \mathrm{~h}, 200$-fold reduction after $8 \mathrm{~h}$, and 400 -fold reduction after $24 \mathrm{~h}$ (Fig. 4a). Therefore, starvation for isoleucine alone is cytocidal for Ca. albicans and partly explains the Ca. albicans ilv2s starvation-cidal phenotype.

We next investigated the individual effect of leucine starvation on the ilv2 $\Delta$ cidal phenotype. Since S. cerevisiae leu 2 mutants die upon leucine starvation (50\% reduction after 2 days) in a different background containing at least one additional auxotrophy (Boer et al., 2008), the viability of S. cerevisiae leu1s (strain S3782, accumulates $\alpha$ isopropylmalate), leu2A (strain S3807, accumulates $\beta$ isopropylmalate) and leu4s leu9s (strain S3783, no 
(a) Recovery on YPD following starvation in SD

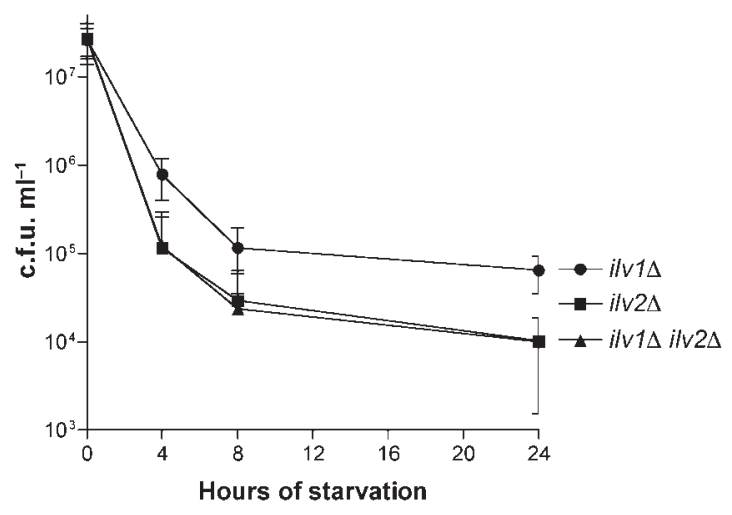

(c) Recovery on YPD or YPEG as indicated following starvation in SD

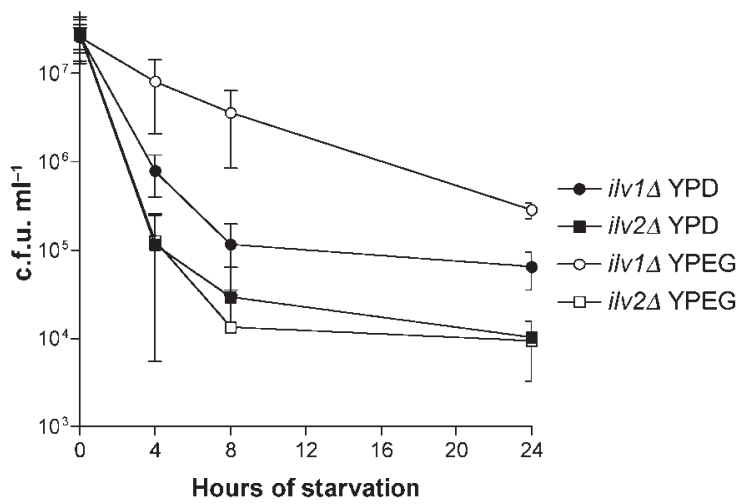

(b) Recovery on YPD following starvation in SD supplemented with amino acids as indicated

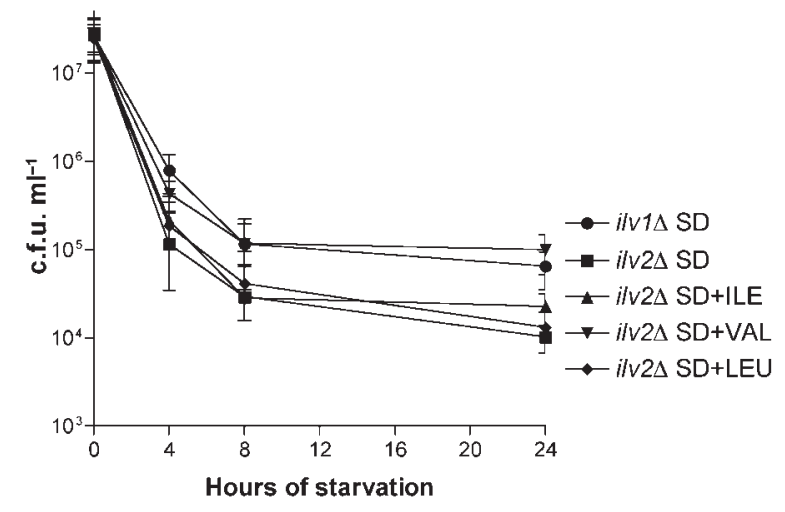

(d) Recovery on YPD following starvation in SD, SD+Rapamycin (Rap) or SEG as indicated

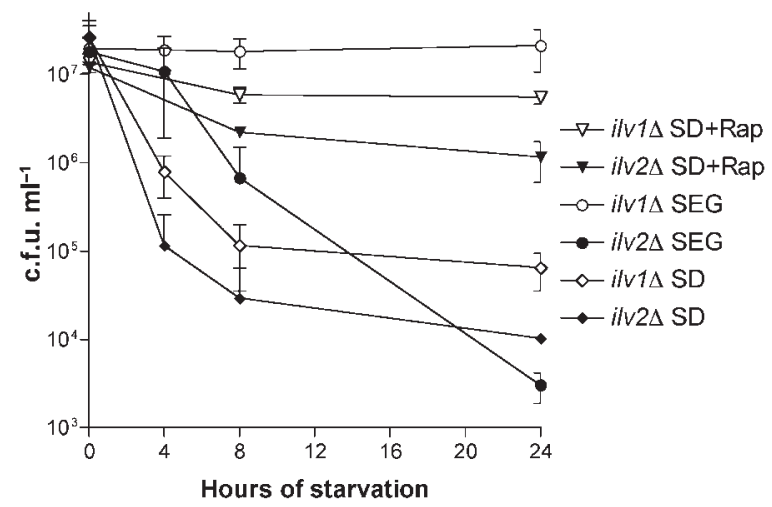

Fig. 4. Influence of genotype, amino acid addition, carbon source present during recovery and starvation, and rapamycin on the

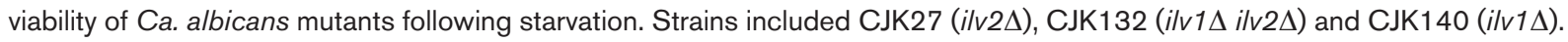

intermediate accumulation) leucine auxotrophs was determined following leucine starvation. Following incubation

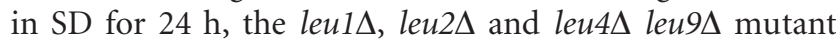
viability was 103,84 and $90 \%$ of input levels, respectively (Fig. 3a). Therefore, in this strain background and experimental protocol, S. cerevisiae leucine starvation results in little to no reduction in viability over a short $(24 \mathrm{~h})$ period. Furthermore, leucine supplementation of starvation media, which results in isoleucine and valine starvation alone, did not alleviate $S$. cerevisiae ilv2 $2 \Delta$ survival, and had little to no effect on Ca. albicans ilv2s survival (Figs $3 \mathrm{~b}$ and $4 \mathrm{~b}$ ). Therefore, in both species, leucine starvation alone also does not explain the ilv2 $\Delta$ cidal phenotypes. Rather, valine starvation alone (both species) or together with isoleucine starvation ( $\mathrm{Ca}$. albicans only) can account for most, if not all, of the starvationcidal phenotypes.

Finally, we assessed the effect of valine starvation. Since the leucine auxotrophy can also be satisfied by valine due to the transamination of valine to the valine and leucine intermediate $\alpha$-ketoisovalerate, valine supplementation results in isoleucine starvation alone (Fig. 1). Although the lack of an effect of isoleucine or leucine starvation alone, as described above, implied that the ilv2 $\Delta$ starvationcidal phenotype was due to valine starvation, valine supplementation did not alleviate the S. cerevisiae ilv2A survival defect during starvation (Fig. 3b). The lack of an effect of valine supplementation may be due to poor uptake of valine by the ilv2 $2 \Delta$ strain in these conditions, such that cells continue to experience a certain state of valine starvation. Consistent with this hypothesis, $S$. cerevisiae ilv2 $2 \Delta$ mutants grew more slowly than the wildtype and ilv1s mutants in both YPD and SD media supplemented with isoleucine and valine (Supplementary Fig. S2). In contrast to $S$. cerevisiae ilv2 $\Delta$ mutants, valine supplementation significantly increased survival following starvation for the Ca. albicans ilv2 $2 \Delta$ mutant at each time point, with survival kinetics more closely resembling those of the Ca. albicans ilv1s mutant (Fig. 4b). Results therefore indicate a significant role for valine starvation in the $S$. cerevisiae and $\mathrm{Ca}$. albicans ilv2 $2 \Delta$ starvation-cidal phenotype. 
Since both isoleucine and valine starvation appeared to be more cytocidal for $\mathrm{Ca}$. albicans than $\mathrm{S}$. cerevisiae, to determine whether $C a$. albicans is more sensitive to amino acid starvation in general than S. cerevisiae, the viability of

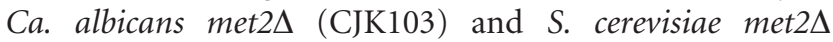
(YJK564) methionine auxotrophs was determined following starvation for $24 \mathrm{~h}$ in medium lacking methionine (SD). After $24 \mathrm{~h}$ starvation, the S. cerevisiae and $\mathrm{Ca}$. albicans met $2 \Delta$ strains were at 116 and $115 \%$ of input levels, respectively (Fig. 3a). Therefore, rather than being a general consequence of amino acid starvation in $\mathrm{Ca}$. albicans, the significantly reduced viability following starvation of Ca. albicans ilv $1 \Delta$ and ilv2 $2 \Delta$ mutants is a unique feature of these mutants.

\section{Recovery from, and sensitivity to, isoleucine and valine starvation is highly influenced by carbon source and rapamycin}

Extreme differences were observed in the colony size of $S$. cerevisiae ilv $2 \Delta$ mutants recovering from isoleucine and valine starvation, which were reminiscent of petite formation (Fig. 5). Since Ca. albicans is generally considered petite-negative (Bulder, 1964), the hypothetical induction of petite formation by isoleucine and valine starvation might explain why $S$. cerevisiae ilv2 $\Delta$ mutants survive this stress at orders of magnitude higher than $\mathrm{Ca}$. albicans ilv $2 \Delta$ mutants. To test whether starvation induced petite formation in S. cerevisiae ilv2 $2 \Delta$ mutants, recovery from starvation was compared on YPD and YPEG. Surprisingly, instead of resulting in a decreased recovery on YPEG plates compared with YPD plates, consistent with petite formation, we observed considerably increased recovery, with c.f.u. approximately the same as input levels on YPEG plates (Fig. 3b). Therefore, rather than dying, $S$. cerevisiae ilv $2 \Delta$ mutants enter into a stage upon isoleucine and valine starvation that is technically viable, but nonrecoverable on YPD.

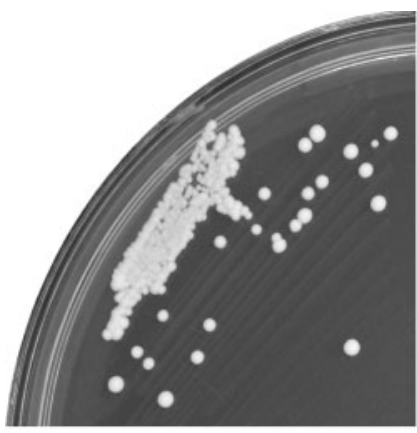

o h starvation

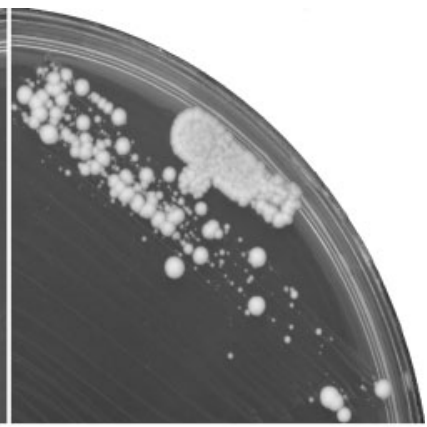

24 h starvation
Fig. 5. Effect of starvation on S. cerevisiae ilv2 $\Delta$ colony size. Cells were starved in SD medium and streaked onto YPD plates at the times indicated.
In contrast to $S$. cerevisiae ilv2 $\Delta$ results, little difference (less than twofold) was observed for recovery of $\mathrm{Ca}$. albicans ilv2s mutants on YPEG compared with YPD following starvation, although a minor improvement (two- to threefold) in recovery was observed for cultures that had been supplemented with valine during starvation (Fig. 4c). However, similar to $S$. cerevisiae ilv2 2 results, we found increased recovery on YPEG compared with YPD following the amino acid starvation of $C a$. albicans ilv1s, particularly at earlier time points, with approximately 10 - and 30 -fold improvements in recovery at 4 and $8 \mathrm{~h}$, respectively (Fig. 4c). Therefore, a proportion of Ca. albicans ilv $1 \Delta$ mutants also appear to enter into a state that is viable, but nonrecoverable on YPD.

Viability loss upon starvation for certain nutrients has been attributed to an inability to enter into a rapid and safe cellcycle arrest upon starvation (Boer et al., 2008). Since this entry is regulated by the carbon source (Gray et al., 2004; Schneper et al., 2004), the carbon source present during nutrient starvation can influence viability (inhibition of arrest in the presence of glucose, arrest in the presence of ethanol and glycerol) (Boer et al., 2008). To investigate whether the carbon source present influences the isoleucine and valine starvation-cidal phenotype, we compared the survival of mutants in SD and SEG starvation media, with or without isoleucine, valine or leucine supplementation. The c.f.u. of $S$. cerevisiae ilv2 $2 \Delta$ and ilv $1 \Delta$ ilv $2 \Delta$ mutants recovered on YPD following starvation in SEG were no higher than when starved in SD, while numbers recovered on YPEG were again at input levels (Fig. 3b). Therefore, the carbon source present during starvation did not influence S. cerevisiae ilv $2 \Delta$ mutant survival.

Again contrasting with S. cerevisiae results, we found that survival of the Ca. albicans ilv2 $2 \Delta$ mutant was significantly enhanced at earlier time points when starved in SEG compared with SD with the respective amino acid supplementations; for example, the average increases in survival in SEG, SEG + isoleucine, SEG + valine and SEG + leucine incubation at $4 \mathrm{~h}$, were 90-, 37-, 31- and 84 -fold, respectively. However, levels surviving more closely approximated those observed for starvation in SD supplemented with the respective amino acids after $24 \mathrm{~h}$ incubation (Fig. 4d includes SEG results, other results not shown). Furthermore, starvation in SEG was completely cytostatic for the ilv1s mutant after $24 \mathrm{~h}$ (Fig. 4d). Therefore, results are consistent with a role for the lack of an orderly cell cycle arrest, particularly in the isoleucine starvation-cidal phenotype in $\mathrm{Ca}$. albicans.

Since the TOR (target of rapamycin) pathway inhibits entry into the quiescent state and the cessation of glucose fermentation in nutrient-rich conditions (Gray et al., 2004; Zaman et al., 2008), we investigated whether starvation in the presence of rapamycin influenced the viability of the $S$. cerevisiae ilv $2 \Delta$ and Ca. albicans ilv $1 \Delta$ and ilv $2 \Delta$ mutants. Rapamycin was added to SD at a concentration of $100 \mathrm{nM}$, since this was the concentration used in starvation assays 
by Boer et al. (2008); this is the $\mathrm{MIC}_{80}$ for wild-type $S$. cerevisiae (S094) and Ca. albicans (SC5314), and is not fungicidal at this concentration (data not shown). Starvation in $\mathrm{SD}+$ rapamycin $(100 \mathrm{nM})$ significantly reduced the recovery of $S$. cerevisiae ilv $2 \Delta$ mutants, with an average 54fold lower recovery on YPD compared with the no-drug control, and an approximately 2000-fold overall decrease after $24 \mathrm{~h}$ (Fig. 3c). In contrast, starvation in SD + rapamycin suppressed the starvation-cidal phenotype of $\mathrm{Ca}$. albicans $i l v 1 \Delta$ mutants, with an average sixfold and 76-fold higher recovery following 8 and $24 \mathrm{~h}$ starvation, respectively, compared with the no-drug control (Fig. 4d). Rapamycin also improved $\mathrm{Ca}$. albicans ilv2 $2 \Delta$ survival, with average recovery rates fivefold (after $8 \mathrm{~h}$ starvation) and 10-fold (after $24 \mathrm{~h}$ starvation) higher than that of the no-drug control. These results provide further support for the lack of an orderly arrest of the cell cycle contributing to the starvationcidal phenotype in Ca. albicans ilv $1 \Delta$ and ilv2 $2 \Delta$ mutants.

\section{DISCUSSION}

Our previous demonstration that S. cerevisiae and $\mathrm{Cr}$. neoformans ilv2 mutants are starvation-cidal and are unable to survive in vivo and/or are avirulent (Kingsbury et al., 2004a, 2006) provided promise for the exploitation of fungal acetolactate synthase as a novel antifungal drug target. In this study, we further strengthen the drug target utility of this enzyme by determining that $\mathrm{Ca}$. albicans ilv2 $2 \Delta$ mutants die rapidly and at profoundly high levels upon isoleucine and valine starvation, and are highly attenuated in virulence.

The highly amino acid starvation-cidal phenotype of $\mathrm{Ca}$. albicans ilv2 $2 \Delta$ mutants indicates that inhibition of Ilv2p in amino acid-limited environments such as in vivo should be fungicidal, rather than fungistatic. Numerous herbicides belonging to three drug classes have already been identified that inhibit acetolactate synthase (Whitcomb, 1999). While inhibitors of the Ca. albicans leucine biosynthetic pathway alone would have little utility in vivo, as leucine auxotrophs are virulent (Kirsch \& Whitney, 1991; Noble \& Johnson, $2005)$, since the toxic effects in ilv2 $\Delta$ mutants are likely to be due to isoleucine and valine starvation, inhibitors of other isoleucine and valine biosynthetic enzymes would likely also be effective. Furthermore, given that isoleucine starvation is also fungicidal and that the ilv1s mutant is somewhat attenuated in virulence in $\mathrm{Ca}$. albicans, any inhibitor targeting an isoleucine biosynthetic enzyme should have a clinically beneficial interaction with any of the various isoleucyl tRNA synthetase inhibitors currently available with antifungal activity, such as the antibacterial agent mupirocin (Nicholas et al., 1999) or the anticandidal drug BAY 10-8888 (PLD-118) (Ziegelbauer, 1998; Ziegelbauer et al., 1998). Finally, since the growth rate of $i l v 2 \Delta$ mutants was reduced compared with the wild-type even in the presence of abundant levels of isoleucine and valine, Ilv2p inhibition may not require complete absence of isoleucine and valine from the environment to have a therapeutic benefit.
We considered the hypothesis that the starvation-cidal phenotypes of fungal ilv2 $2 \Delta$ mutants were due to accumulation of the biosynthetic intermediate $\alpha$-ketobutyrate, since $\alpha$-ketobutyrate or its transamination product, $\alpha$ aminobutyric acid, accumulates upon inhibition of acetolactate synthase in plants and bacteria (LaRossa et al., 1987; Rhodes et al., 1987; Shaner \& Singh, 1993). Although results from other researchers have shown a lack of correlation between $\alpha$-ketobutyrate accumulation and growth inhibition (Epelbaum et al., 1996; Landstein et al., 1990; Shaner \& Singh, 1993), the high potency associated with the inhibition of acetolactate synthase has often been attributed to $\alpha$-ketobutyrate toxicity (Daniel et al., 1983, 1984; LaRossa \& Van Dyk, 1987; LaRossa et al., 1987; Rhodes et al., 1987; Van Dyk et al., 1987). However, since neither $S$. cerevisiae nor Ca. albicans ilv2 $2 \Delta$ mutants were sensitive to high exogenous levels of $\alpha$-ketobutyrate, and ilv1s ilv2 $2 \Delta$ mutants that cannot accumulate $\alpha$ ketobutyrate were as starvation-cidal as ilv $2 \Delta$ mutants, we ruled out the $\alpha$-ketobutyrate accumulation hypothesis (Epelbaum et al., 1996) as the explanation for the $S$. cerevisiae and Ca. albicans ilv2 $2 \Delta$ starvation-cidal phenotypes.

Interestingly, isoleucine-auxotrophic Ca. albicans ilv1s mutants were also starvation-cidal, while $S$. cerevisiae ilv1 $\Delta$ mutants were starvation-static over the same time period; thus, isoleucine starvation is more deleterious in $C a$. albicans than in $S$. cerevisiae. The more severe starvation-cidal phenotype in ilv2 $2 \Delta$ mutants compared with ilv1 $1 \Delta$ mutants in both species and leucine auxotrophs in $S$. cerevisiae, together with the 10 -fold increased survival upon supplementation of $C a$. albicans ilv2 $2 \Delta$ mutants with valine during starvation, but not isoleucine or leucine, suggest that valine starvation is more deleterious than isoleucine or leucine starvation in both species.

The carbon source present during recovery from starvation had a major effect on recovery from starvation of $S$. cerevisiae ilv $2 \Delta$ and $C a$. albicans $i l v 1 \Delta$ mutants, with greatly enhanced recovery when ethanol and glycerol were the carbon sources compared with glucose. The carbon sourcedependent recovery is reminiscent of the viable but nonculturable phenomenon explored extensively in bacteria, whereby following exposure to various stresses such as starvation, cells that are metabolically active fail to grow under classical culture conditions, but may be able to be resuscitated upon administration of a certain trigger (Kell et al., 1998; Oliver, 2005). An analogous state has also been recorded for Saccharomyces, Candida and other yeast species following alcoholic fermentation and $\mathrm{SO}_{2}$ addition during wine production (Divol \& Lonvaud-Funel, 2005; Mills et al., 2002).

In contrast to $S$. cerevisiae ilv $2 \Delta$ mutants, viability following starvation of $C a$. albicans ilv $1 \Delta$ and ilv2 $2 \Delta$ mutants was strongly influenced by the carbon source present during starvation, with enhanced survival when mutants were incubated in ethanol and glycerol compared with glucose. 
Further contrasting with $S$. cerevisiae ilv $2 \Delta$ mutants, in which rapamycin reduced viability upon starvation, we observed a suppression of cell death upon starvation of both $C a$. albicans ilv $1 \Delta$ and ilv2 $2 \Delta$ mutants when starved in the presence of rapamycin, an inhibitor of the TOR pathway that controls entry into stationary phase and cessation of glucose utilization when nutrients are plentiful (Gray et al., 2004; Zaman et al., 2008). Taken together, these $\mathrm{Ca}$. albicans results are similar to the findings of Boer et al. (2008), who proposed that since glucose represses pathways that activate entry into a resting state (Gray et al., 2004; Schneper et al., 2004), the cells are failing to undergo a rapid and prompt cell arrest when glucose is the carbon source, and are wasting glucose, analogous to the Warburg effect described in tumours (Warburg, 1956).

Since $S$. cerevisiae met $2 \Delta$ and $C a$. albicans met $2 \Delta$ mutants were equally starvation-static upon methionine starvation, amino acid starvation is not generally more starvationcidal in Ca. albicans than S. cerevisiae. Therefore, two questions remain: first, precisely why ilv $2 \Delta$ mutants are starvation-cidal; and second, why $C a$. albicans ilv2 $\Delta$ mutants die, or fail to recover, from isoleucine and valine starvation at such a rapid rate and to a significantly higher degree than Cr. neoformans and S. cerevisiae ilv $2 \Delta$ mutants. Possible mechanisms may involve differences in the levels of misincorporation of other amino acids into proteins in the absence of valine and/or isoleucine and leucine, or differences in the timing or degree of cellular arrest upon starvation. The effect of rapamycin on the ilv2 $2 \Delta$ starvation phenotype indicates an intimate association of the TOR pathway with this phenotype in both species, and the species-specific differences in the rapamycin response and starvation severity may be consequences of differences in the wiring of the TOR pathway between species. Further research is required to better understand both the speciesspecific differences in the rapamycin response and starvation severity, as well as why in both species, starvation for one amino acid, such as methionine, is static, while starvation for others, such as isoleucine and valine, is cidal.

\section{ACKNOWLEDGEMENTS}

The authors would like to thank Dr Joseph Heitman and Dr Maria Cardenas-Corona for the critical evaluation of this manuscript, and Dr Heitman's laboratory (Duke University Medical Center) for the gift of the plasmid pSFS2A, with permission from Dr Joachim Morschhäuser. This study was funded by the National Institutes of Health R21 grant AI070247.

\section{REFERENCES}

Barclay, B. J. \& Little, J. G. (1977). Selection of yeast auxotrophs by thymidylate starvation. J Bacteriol 132, 1036-1037.

Boer, V. M., Amini, S. \& Botstein, D. (2008). Influence of genotype and nutrition on survival and metabolism of starving yeast. Proc Natl Acad Sci U S A 105, 6930-6935.
Brauer, M. J., Huttenhower, C., Airoldi, E. M., Rosenstein, R., Matese, J. C., Gresham, D., Boer, V. M., Troyanskaya, O. G. \& Botstein, D. (2008). Coordination of growth rate, cell cycle, stress response, and metabolic activity in yeast. Mol Biol Cell 19, 352-367.

Bulder, C. J. (1964). Induction of petite mutation and inhibition of synthesis of respiratory enzymes in various yeasts. Antonie Van Leeuwenhoek 30, 1-9.

Chipman, D., Barak, Z. \& Schloss, J. V. (1998). Biosynthesis of 2aceto-2-hydroxy acids: acetolactate synthases and acetohydroxyacid synthases. Biochim Biophys Acta 1385, 401-419.

Crispens, C. G. (1975). Section IV. Blood. In Handbook on the Laboratory Mouse, pp. 93-123. Springfield, IL: Charles C. Thomas.

Culbertson, M. R. \& Henry, S. A. (1975). Inositol-requiring mutants of Saccharomyces cerevisiae. Genetics 80, 23-40.

Cynober, L. A. (2002). Plasma amino acid levels with a note on membrane transport: characteristics, regulation, and metabolic significance. Nutrition 18, 761-766.

Daniel, J., Dondon, L. \& Danchin, A. (1983). 2-Ketobutyrate: a putative alarmone of Escherichia coli. Mol Gen Genet 190, 452-458.

Daniel, J., Joseph, E. \& Danchin, A. (1984). Role of 2-ketobutyrate as an alarmone in E. coli K12: inhibition of adenylate cyclase activity mediated by the phosphoenolpyruvate: glycose phosphotransferase transport system. Mol Gen Genet 193, 467-472.

Divol, B. \& Lonvaud-Funel, A. (2005). Evidence for viable but nonculturable yeasts in botrytis-affected wine. J Appl Microbiol 99, 85-93.

Epelbaum, S., Chipman, D. M. \& Barak, Z. (1996). Metabolic effects of inhibitors of two enzymes of the branched-chain amino acid pathway in Salmonella typhimurium. J Bacteriol 178, 1187-1196.

Fradin, C., Kretschmar, M., Nichterlein, T., Gaillardin, C., d'Enfert, C. \& Hube, B. (2003). Stage-specific gene expression of Candida albicans in human blood. Mol Microbiol 47, 1523-1543.

Fradin, C., De Groot, P., MacCallum, D., Schaller, M., Klis, F., Odds, F. C. \& Hube, B. (2005). Granulocytes govern the transcriptional response, morphology and proliferation of Candida albicans in human blood. Mol Microbiol 56, 397-415.

Gillum, A. M., Tsay, E. Y. \& Kirsch, D. R. (1984). Isolation of the Candida albicans gene for orotidine- 5 '-phosphate decarboxylase by complementation of S. cerevisiae ura3 and E. coli pyrF mutations. Mol Gen Genet 198, 179-182.

Goldstein, A. L. \& McCusker, J. H. (1999). Three new dominant drug resistance cassettes for gene disruption in Saccharomyces cerevisiae. Yeast 15, 1541-1553.

Goldstein, A. L. \& McCusker, J. H. (2001). Development of Saccharomyces cerevisiae as a model pathogen. A system for the genetic identification of gene products required for survival in the mammalian host environment. Genetics 159, 499-513.

Gray, J. V., Petsko, G. A., Johnston, G. C., Ringe, D., Singer, R. A. \& Werner-Washburne, M. (2004). "Sleeping beauty": quiescence in Saccharomyces cerevisiae. Microbiol Mol Biol Rev 68, 187-206.

Guldener, U., Heck, S., Fielder, T., Beinhauer, J. \& Hegemann, J. H. (1996). A new efficient gene disruption cassette for repeated use in budding yeast. Nucleic Acids Res 24, 2519-2524.

Henry, S. A., Donahue, T. F. \& Culbertson, M. R. (1975). Selection of spontaneous mutants by inositol starvation in yeast. Mol Gen Genet 143, 5-11.

Hoffman, C. S. \& Winston, F. (1987). A ten-minute DNA preparation from yeast efficiently releases autonomous plasmids for transformation of Escherichia coli. Gene 57, 267-272.

Ito-Harashima, S., Hartzog, P. E., Sinha, H. \& McCusker, J. H. (2002). The tRNA-Tyr gene family of Saccharomyces cerevisiae: agents of 
phenotypic variation and position effects on mutation frequency. Genetics 161, 1395-1410.

Kell, D. B., Kaprelyants, A. S., Weichart, D. H., Harwood, C. R. \& Barer, M. R. (1998). Viability and activity in readily culturable bacteria: a review and discussion of the practical issues. Antonie Van Leeuwenhoek 73, 169-187.

Kingsbury, J. M. \& McCusker, J. H. (2008). Threonine biosynthetic genes are essential in Cryptococcus neoformans. Microbiology 154, 2767-2775.

Kingsbury, J. M., Yang, Z., Ganous, T. M., Cox, G. M. \& McCusker, J. H. (2004a). Cryptococcus neoformans Ilv2p confers resistance to sulfometuron methyl and is required for survival at $37^{\circ} \mathrm{C}$ and in vivo. Microbiology 150, 1547-1558.

Kingsbury, J. M., Yang, Z., Ganous, T. M., Cox, G. M. \& McCusker, J. H. (2004b). A novel chimeric spermidine synthase-saccharopine dehydrogenase (SPE3-LYS9) gene in the human pathogen Cryptococcus neoformans. Eukaryot Cell 3, 752-763.

Kingsbury, J. M., Goldstein, A. L. \& McCusker, J. H. (2006). Role of nitrogen and carbon transport, regulation, and metabolism genes for Saccharomyces cerevisiae survival in vivo. Eukaryot Cell 5, 816-824.

Kirsch, D. R. \& Whitney, R. R. (1991). Pathogenicity of Candida albicans auxotrophic mutants in experimental infections. Infect Immun 59, 3297-3300.

Landstein, D., Chipman, D. M., Arad, S. M. \& Barak, Z. (1990). Acetohydroxy acid synthase activity in Chlorella emersonii under auto- and heterotrophic growth conditions. Plant Physiol 94, 614620.

LaRossa, R. A. \& Van Dyk, T. K. (1987). Metabolic mayhem caused by 2-ketoacid imbalances. Bioessays 7, 125-130.

LaRossa, R. A., Van Dyk, T. K. \& Smulski, D. R. (1987). Toxic accumulation of $\alpha$-ketobutyrate caused by inhibition of the branchedchain amino acid biosynthetic enzyme acetolactate synthase in Salmonella typhimurium. J Bacteriol 169, 1372-1378.

Liebmann, B., Muhleisen, T. W., Muller, M., Hecht, M., Weidner, G., Braun, A., Brock, M. \& Brakhage, A. A. (2004). Deletion of the Aspergillus fumigatus lysine biosynthesis gene lys $F$ encoding homoaconitase leads to attenuated virulence in a low-dose mouse infection model of invasive aspergillosis. Arch Microbiol 181, 378-383.

Lorenz, M. C., Bender, J. A. \& Fink, G. R. (2004). Transcriptional response of Candida albicans upon internalization by macrophages. Eukaryot Cell 3, 1076-1087.

McCusker, J. H., Clemons, K. V., Stevens, D. A. \& Davis, R. W. (1994). Genetic characterization of pathogenic Saccharomyces cerevisiae isolates. Genetics 136, 1261-1269.

Mills, D. A., Johannsen, E. A. \& Cocolin, L. (2002). Yeast diversity and persistence in Botrytis-affected wine fermentations. Appl Environ Microbiol 68, 4884-4893.

Nazi, I., Scott, A., Sham, A., Rossi, L., Williamson, P. R., Kronstad, J. W. \& Wright, G. D. (2007). Role of homoserine transacetylase as a new target for antifungal agents. Antimicrob Agents Chemother 51, 1731-1736.

Nicholas, R. O., Berry, V., Hunter, P. A. \& Kelly, J. A. (1999). The antifungal activity of mupirocin. J Antimicrob Chemother 43, 579-582.

Noble, S. M. \& Johnson, A. D. (2005). Strains and strategies for largescale gene deletion studies of the diploid human fungal pathogen Candida albicans. Eukaryot Cell 4, 298-309.
Oliver, J. D. (2005). The viable but nonculturable state in bacteria. J Microbiol 43 (special issue), 93-100.

Pascon, R. C., Ganous, T. M., Kingsbury, J. M., Cox, G. M. \& McCusker, J. H. (2004). Cryptococcus neoformans methionine synthase: expression analysis and requirement for virulence. Microbiology 150, 3013-3023.

Reuss, O., Vik, A., Kolter, R. \& Morschhauser, J. (2004). The SAT1 flipper, an optimized tool for gene disruption in Candida albicans. Gene 341, 119-127.

Rhodes, D., Hogan, A. L., Deal, L., Jamieson, G. C. \& Haworth, P. (1987). Amino acid metabolism of Lemna minor L.: II. Responses to chlorsulfuron. Plant Physiol 84, 775-780.

Saldanha, A. J., Brauer, M. J. \& Botstein, D. (2004). Nutritional homeostasis in batch and steady-state culture of yeast. Mol Biol Cell 15, 4089-4104.

Sambrook, J., Fritsch, E. F. \& Maniatis, T. (1989). Molecular Cloning: a Laboratory Manual. Cold Spring Harbor, NY: Cold Spring Harbor Laboratory.

Schneper, L., Duvel, K. \& Broach, J. R. (2004). Sense and sensibility: nutritional response and signal integration in yeast. Curr Opin Microbiol 7, 624-630.

Shaner, D. L. \& Singh, B. K. (1993). Phytotoxicity of acetohydroxyacid synthase inhibitors is not due to accumulation of 2-ketobutyrate and/or 2-aminobutyrate. Plant Physiol 103, 1221-1226.

Sherman, F., Fink, G. R. \& Lawrence, C. W. (1974). Methods in Yeast Genetics. Cold Spring Harbor, NY: Cold Spring Harbor Laboratory.

Tkacz, J. S. \& DiDomenico, B. (2001). Antifungals: what's in the pipeline. Curr Opin Microbiol 4, 540-545.

Unger, M. W. \& Hartwell, L. H. (1976). Control of cell division in Saccharomyces cerevisiae by methionyl-tRNA. Proc Natl Acad Sci U S A 73, 1664-1668.

Van Dyk, T. K., Smulski, D. R. \& Chang, Y. Y. (1987). Pleiotropic effects of poxA regulatory mutations of Escherichia coli and Salmonella typhimurium, mutations conferring sulfometuron methyl and $\alpha$ ketobutyrate hypersensitivity. J Bacteriol 169, 4540-4546.

Wach, A., Brachat, A., Pohlmann, R. \& Philippsen, P. (1994). New heterologous modules for classical or PCR-based gene disruptions in Saccharomyces cerevisiae. Yeast 10, 1793-1808.

Warburg, O. (1956). On the origin of cancer cells. Science 123, 309314.

Whitcomb, C. E. (1999). An introduction to ALS-inhibiting herbicides. Toxicol Ind Health 15, 231-239.

Yang, Z., Pascon, R. C., Alspaugh, A., Cox, G. M. \& McCusker, J. H. (2002). Molecular and genetic analysis of the Cryptococcus neoformans MET3 gene and a met3 mutant. Microbiology 148, 2617-2625.

Zaman, S., Lippman, S. I., Zhao, X. \& Broach, J. R. (2008). How Saccharomyces responds to nutrients. Annu Rev Genet 42, 27-81.

Ziegelbauer, K. (1998). Decreased accumulation or increased isoleucyl-tRNA synthetase activity confers resistance to the cyclic $\beta$ amino acid BAY 10-8888 in Candida albicans and Candida tropicalis. Antimicrob Agents Chemother 42, 1581-1586.

Ziegelbauer, K., Babczinski, P. \& Schonfeld, W. (1998). Molecular mode of action of the antifungal $\beta$-amino acid BAY $10-8888$. Antimicrob Agents Chemother 42, 2197-2205.

Edited by: J. Pla 